\title{
Extensive bilateral intracranial calcifications and seizure in iatrogenic hypoparathyroidism: A case report
}

\author{
Parikshit Chapagain $^{1}$, Shambhu Khanal ${ }^{2}$, Rajeev Ojha ${ }^{1}$, Niraj Gautam ${ }^{1}$, Matina Sayami ${ }^{1}$, \\ and Roshan Bhandari ${ }^{1}$ \\ ${ }^{1}$ Tribhuvan University Teaching Hospital \\ ${ }^{2}$ Tribhuvan University Institute of Medicine
}

July 19, 2021

\begin{abstract}
Calcification beyond basal ganglia is rare in postoperative permanent hypoparathyroidism. We report extensive bilateral intracranial calcifications involving basal ganglia, thalamus, cerebellum, and cerebral cortex in a 56-year-old lady who presented with carpopedal spasm, seizure, and severe hypocalcemia after 20 years of near-total thyroidectomy.
\end{abstract}

\section{INTRODUCTION}

Postoperative permanent hypoparathyroidism is characterized by hypocalcemia, hyperphosphatemia, and low to inappropriately normal parathyroid hormone levels, which persist more than six months after surgery. ${ }^{1}$ It occurs in $0.3 \%$ to $5.1 \%$ of individuals who undergo thyroid surgery for thyroid cancer. ${ }^{2-4}$ Severe hypocalcemia can present with carpopedal spasms, tetany, seizures, and cardiac dysrhythmias. Longstanding postoperative hypoparathyroidism is characterized by intracranial calcifications of basal ganglia and cerebellum; calcifications beyond these areas are rare. ${ }^{1,5}$ We report the case of a 56-year-old lady who presented with carpopedal spasms, tetany, generalized tonic-clonic seizure, and extensive bilateral brain calcifications due to postoperative permanent hypoparathyroidism.

\section{CASE REPORT}

A normotensive, nondiabetic 56-year-old lady, who had undergone near-total thyroidectomy for papillary carcinoma of thyroid 20 years ago, presented in the emergency department with history of spasms in her hands and calves, followed by sustained contraction of hand muscles. Then she had an episode of tonic-clonic generalized abnormal body movements with uprolling of eyes and frothing from mouth, which lasted about 30-35 seconds; it was followed by a brief period of confusion and amnesia. She had been taking calcium, vitamin $\mathrm{D}$, and hormonal supplements for hypothyroidism after the surgery until five months ago when she stopped taking them due to her general good condition. Four months ago, she experienced episodes of calf spasms, which lasted about five minutes and were severe enough to limit walking. On seeking medical care, the episodes were attributed to calcium deficiency, and she was prescribed calcium and cholecalciferol. She had undergone cataract surgery eight years ago. She had no history of fever, headache, vomiting, palpitation, or chest pain.

On examination, the patient had positive trousseau's sign on tourniquet test, which manifested as carpopedal spasm: flexion of the wrist and metacarpophalangeal joints, extension of the interphalangeal joints, and adduction of the thumb. She had an old, transverse post-surgical scar on her neck. Ophthalmological evaluation revealed an intraocular lens implant in each eye. There was no pallor, icterus, clubbing, or lymphadenopathy. Her respiratory, cardiovascular, abdominal, and neurological examinations were unremarkable. 
Laboratory investigations revealed the following findings: corrected serum total calcium, $1.25 \mathrm{mmol} / \mathrm{L}$ (normal range, 2.1-2.6); serum phosphorus, $2.19 \mathrm{mmol} / \mathrm{L}$ (normal range, 0.8-1.54); Intact Parathyroid Hormone (IPTH), $5.2 \mathrm{ng} / \mathrm{L}$ (normal range, 7.5-53.5); 25-hydroxyvitamin D, $112.07 \mathrm{nmol} / \mathrm{L}$ (normal range, 74.88249.6); magnesium, $0.53 \mathrm{mmol} / \mathrm{L}$ (normal range, 0.69-1.02); serum albumin, $39 \mathrm{~g} / \mathrm{L}$ (normal range, 38-49); Alkaline Phosphatase (ALP), $221 \mathrm{U} / \mathrm{L}$ (normal range, 90-306); free T3, 3.05 pmol/L (normal range, 4.26-8.1); free T4, $28.3 \mathrm{pmol} / \mathrm{L}$ (normal range, 10.2-28.2); Thyroid Stimulating Hormone (TSH), $0.31 \mathrm{mU} / \mathrm{L}$ (normal range, 0.46-4.68); serum sodium, $138 \mathrm{meq} / \mathrm{L}$ (normal range, 135-145); and potassium, $5.1 \mathrm{meq} / \mathrm{L}$ (normal range 3.5-5). Her ECG showed normal sinus rhythm with no QT interval prolongation.

Computed tomography of the brain revealed extensive bilateral calcifications involving basal ganglia, thalami, periventricular region, subcortical fronto-pareito-occipital region, and cerebellum (Figure 1).

The patient was treated with intravenous calcium gluconate, calcitriol, and magnesium. Serum calcium and magnesium levels were checked daily. After four days of intravenous calcium treatment, total calcium rose to $2.1 \mathrm{mmol} / \mathrm{L}$. Her spasms subsided, and seizure did not recur. On the fifth day, intravenous calcium was replaced by oral calcium. She was discharged on $1500 \mathrm{mg}$ of oral calcium and $1 \mathrm{mcg}$ of oral calcitriol daily.

The patient was explained about the appropriate supplements and advised to follow up every three months. She followed up on the third month of discharge. She had strictly adhered to the treatment regimen and had not experienced seizure or spasm again; her total calcium level was $2.15 \mathrm{mmol} / \mathrm{L}$ (normal range, 2.1-2.6).

\section{DISCUSSION}

Hypoparathyroidism is the commonest postoperative complication after thyroid surgery and is characterized by hypocalcemia, elevated serum phosphorus levels, and low or inappropriately normal plasma levels of parathyroid hormone. ${ }^{1,4}$ Postoperative permanent hypoparathyroidism is characterized by the persistence of hypocalcemia beyond six months of surgery. ${ }^{1,6}$ Its incidence depends on the extent of thyroidectomy and lymph node dissection. ${ }^{1-3}$ Similarly, it occurs more frequently after surgeries done for thyroid cancer than those done for benign diseases. ${ }^{4}$

Hypocalcemia presents classically with muscle twitching, spasms, tingling, and numbness. Carpopedal spasm is characteristic and, in severe cases, can progress to tetany, seizures, and cardiac dysrhythmias. Signs of neuromuscular excitability can be observed on provocation tests: Chvostek's sign and Trousseau's sign. ${ }^{7}$ Hypocalcemia associated with chronic hypoparathyroidism exhibits several unique features such as basal ganglia calcifications, neuropsychiatric symptoms, and cataracts. ${ }^{7}$ Calcification of the brain occurs less frequently in postoperative hypoparathyroidism than in other causes of hypoparathyroidism; one reason behind this is believed to be the earlier detection of hypoparathyroidism resulting in earlier supplementation of calcium and Vitamin D following surgery. ${ }^{5,8}$ The calcifications typically involve caudate nucleus, putamen, globus pallidus, thalamus, and dentate nucleus; calcifications beyond these areas are rare. ${ }^{5,9}$ Our patient had extensive bilateral intracranial calcifications, which involved the basal ganglia, thalamus, periventricular region, subcortical fronto-pareito-occipital region, and cerebellum. Some patients with basal ganglia calcifications develop Parkinsonism, while others, like our patient, do not. ${ }^{5,9,10}$ She had also undergone cataract surgery of both eyes eight years ago.

Patients with permanent hypoparathyroidism require life-long calcium supplementation. The goal is to maintain serum calcium in the low normal range, and serum calcium should be checked every three to six months or when the medical regimen is changed. ${ }^{6}$ The synthesis of 1,25 -dihydroxyvitamin $\mathrm{D}$ requires parathyroid hormone; therefore, it is deficient in patients with hypoparathyroidism and needs to be supplemented. Commonly available vitamin D preparations contain ergocalciferol or cholecalciferol, both of which are ineffective in hypoparathyroidism for the reason mentioned above. ${ }^{7}$ Our patient stopped checking her calcium levels one year ago, and due to her general good condition, she stopped taking calcium and vitamin D supplements as well, five months ago. Within a month of stopping the supplements, she experienced carpopedal spasm, which was empirically treated with calcium and cholecalciferol supplements without checking calcium levels. She was not prescribed calcitriol. This probably led to the severe hypocalcemia that she had on presentation. 


\section{CONCLUSION}

Postoperative permanent hypoparathyroidism can lead to extensive bilateral intracranial calcifications involving basal ganglia, thalamus, cerebellum, and cerebral cortex. Severe hypocalcemia can occur even after several years of surgery; therefore, serum calcium levels of individuals with postoperative permanent hypoparathyroidism should be measured life-long at regular intervals. Hypoparathyroidism should be considered in post-thyroidectomy individuals who present with seizures or intracranial calcifications.

\section{LIST OF ABBREVIATIONS}

ALP: Alkaline Phosphatase

ECG: Electrocardiogram

IPTH: Intact Parathyroid Hormone

TSH: Thyroid Stimulating Hormone

\section{Acknowledgements}

The authors are grateful to the patient and her family for co-operating in the preparation of this manuscript.

\section{Conflict of interest}

The authors declare that they have no competing interests.

\section{Author contributions}

PC and SK: wrote the initial draft of the manuscript; RO, NG, MS, and RB: reviewed the manuscript; PC and SK: edited the draft and reshaped it into this manuscript. All authors read and approved the final manuscript.

\section{Ethical approval and consent to participate}

Need for ethical approval waived. Consent from the patient deemed to be enough.

\section{Consent for publication}

Written informed consent was obtained from the patient for publication of this case report and any accompanying images.

\section{REFERENCES}

1. Kakava K, Tournis S, Papadakis G, et al. Postsurgical Hypoparathyroidism: A Systematic Review. In Vivo . 2016;30(3):171-179.

2. Lee YS, Nam K-H, Chung WY, Chang H-S, Park CS. Postoperative Complications of Thyroid Cancer in a Single Center Experience. J Korean Med Sci . 2010;25(4):541-545. doi:10.3346/jkms.2010.25.4.541

3. Filho JG, Kowalski LP. Postoperative complications of thyroidectomy for differentiated thyroid carcinoma. American Journal of Otolaryngology . 2004;25(4):225-230. doi:10.1016/j.amjoto.2004.02.001

4. Rosato L, Avenia N, Bernante P, et al. Complications of Thyroid Surgery: Analysis of a Multicentric Study on 14,934 Patients Operated on in Italy over 5 Years. World J Surg . 2004;28(3):271-276. doi:10.1007/s00268-003-6903-1

5. Posen S. Computerized Tomography of the Brain in Surgical Hypoparathyroidism. Ann Intern Med . 1979;91(3):415. doi:10.7326/0003-4819-91-3-415

6. Schafer AL, Shoback DM. Hypocalcemia: Diagnosis and Treatment . MDText.com, Inc.; 2016. Accessed May 13, 2020.

https://www.ncbi.nlm.nih.gov/books/NBK279022/ 
7. Cooper MS, Gittoes NJL. Diagnosis and management of hypocalcaemia.BMJ . 2008;336(7656):1298-1302. doi:10.1136/bmj.39582.589433.BE

8. Dimich A, Bedrossian PB, Wallach S. Hypoparathyroidism. Clinical observations in 34 patients. Arch Intern Med . 1967;120(4):449-458. doi:10.1001/archinte.120.4.449

9. Agarwal R, Lahiri D, Biswas A, Mukhopadhyay J, Maity P, Roy M. A rare cause of seizures, parkinsonian, and cerebellar signs: Brain calcinosis secondary to thyroidectomy. North Am J Med Sci . 2014;6(10):540. doi:10.4103/1947-2714.143287

10. Frame B. Parkinsonism in Postoperative Hypoparathyroidism.Arch Intern Med . 1965;116(3):424. doi:10.1001/archinte.1965.03870030104017

\section{Figure legend}

Figure 1. Plain computed tomography of the brain. Bilateral calcifications are seen in cerebellum (A, black arrows); basal ganglia (B, black arrowheads); thalami, periventricular region ( $\mathrm{C}$, white arrowheads); and subcortical fronto-pareito-occipital region $(\mathrm{D}$, white arrows)
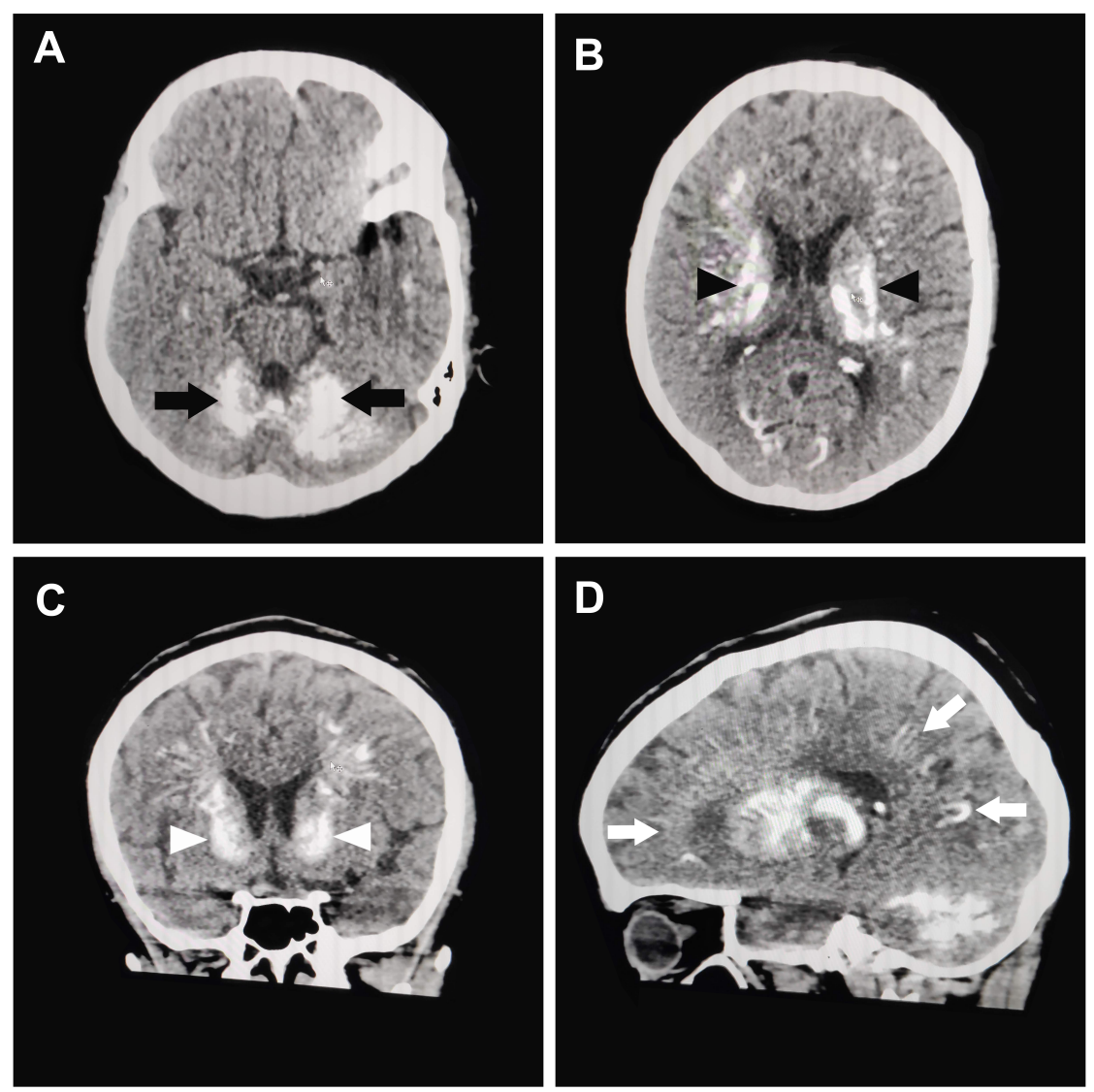